IDDF2019-ABS-0233 LONG TERM INDWELLING TRANSMURAL STENTS IN PATIENTS WITH WALLED OFF PANCREATIC NECROSIS AND DISCONNECTED PANCREATIC DUCT SYNDROME: SAFETY AND EFFICACY

${ }^{1}$ Jimil Shah*, ${ }^{1}$ Surinder Rana, ${ }^{1}$ Ravi Sharma, ${ }^{2}$ Rajesh Gupta. ${ }^{1}$ Department of Gastroenterology, PGIMER, Chandigarh, India; 'Department of Surgery, PGIMER, Chandigarh, India

\subsection{6/gutjnl-2019-IDDFabstracts.204}

Background Long-term indwelling transmural stents in patients with walled-off pancreatic necrosis (WOPN) and disconnected pancreatic duct syndrome (DPDS) has been accepted as an effective strategy to decrease the risk of recurrence of pancreatic fluid collection (PFC). However, long term studies on the safety and efficacy of leaving permanent indwelling plastic prosthesis are lacking.

Methods Retrospective analysis of the database of patients with WOPN treated with endoscopic transmural drainage over the last 8 years was done to identify patients with DPDS and permanently intended indwelling transmural stents. Patients with indwelling stents for more than 3 years were only included in this study. The follow-up database was analysed for any recurrence of symptoms or PFC or complications.

Results During the last 8 years, 179 patients of WOPN were treated with endoscopic transmural drainage and 138 (77.09\%) patients had DPDS. Of 138 patients, 56 patients (39 males; age range: 21-62 years) had an indwelling transmural stent/stents for $>3$ years (3-8 years). Amongst plastic prosthesis, $67.85 \%$ of patients had $10 \mathrm{Fr}$ stents and $32.14 \%$ of patients had 7 Fr stents. Neck $(n=28 ; 50 \%)$ was the most common site of disruption followed by proximal body $(n=16$; $28.57 \%)$ and distal body $(n=12 ; 21.43 \%)$. One patient $(1.78 \%)$ developed recurrence of fluid collection 58 months later despite of in-situ stent which was successfully treated with repeat endoscopic transmural drainage. Two (3.5\%) patients had asymptomatic spontaneous external migration of the transmural stent. Two (3.5\%) patients developed complications 4 years later because of the indwelling stent. The transmural stent eroded into descending colon in one patient and the other patient had a recurrence of abdominal pain due to the development of pancreatic parenchymal calcification around the pigtail of the plastic stent. The transmural stent was removed in both patients and this led to the closure of colonic fistula as well cessation of abdominal pain. No other complication of long term indwelling transmural stents was observed.

Conclusions Long term indwelling transmural stents in patients with WOPN and DPDS are safe as well as effective in preventing recurrence of PFC.

\section{IDDF2019-ABS-0238 FOLLOW-UP OF SMALL BOWEL AND DRY TYPE PERITONEAL TUBERCULOSIS TREATMENT}

Maria Goretti Ametembun*. Internal Medicine Clinic St Elisabeth Hospital, Lela, Sikka District, Flores - Indonesia, Indonesia

\subsection{6/gutjnl-2019-IDDFabstracts.205}

Background The aim of this study was to describe the followup of the small bowel and dry type peritoneal tuberculosis treatment.
Methods This cross-sectional study was conducted at i St Elisabeth Hospital, Flores, Nusa Tenggara Timur from February 2012 through August 2013 by one internal medicine specialist who has certificate of ultrasound, including sign and symptoms, laboratory, thorax X-ray and also abdominal ultrasound examination on tympanic and dullness area according to 'dam board phenomenon' of dry type peritoneal tuberculosis.

Results $\mathrm{N}=546$ (14-85 y, mean 36,5 y), 312(57\%) female, 234(43\%) male. Preceding treatment: All patients had constitutional symptoms as general discomfort, fatigue, loss of appetite, weight loss, low-grade fever and night sweating, chronic recurrent cough, positive thorax X-ray, all with negative microscopic smear and all negative HIV test except 1 patient. The symptoms \& signs were recurrent colic abdomen, diarrhoea or constipation, abdominal distention, doughy abdomen, "Dam-board' phenomenon". Ultrasound findings were the thickness of small bowel wall in the dullness pain area were more than in tympanic normal area. Furthermore hypo-peristaltic, differentiation of wall layers was lost, and irregular margin of small bowel wall and narrowing of the lumen were found in the affected dullness area. No Mycobacterium microscopic smear/culture from sputum/feces nor small bowel biopsy were not available. During the 9-12 months of the anti-tuberculosis treatment period, the symptoms and signs both constitutional and abdominal were decreased gradually. Dullness area according to dam-board phenomenon disappeared gradually as well as and small bowel wall thickening and lumen narrowing at the affected area. After the treatment: no constitutional sign \&symptoms, no colic abdominal pain, no more tenderness, no doughy abdomen nor dullness area anymore. Only $292(53 \%)$ patients then carried out a re-examination of ultrasound at the end of treatment (due to economic reason) showed small bowel wall thickening were decreased and normal peristaltic movement was seemed on the affected area, otherwise the margin of the small bowel wall was still irregular.

Conclusions Anti-tuberculosis for 9-12 months was effectively to treat small bowel and peritoneal tuberculosis dry type.

\section{IDDF2019-ABS-0239 CLINICAL OUTCOMES OF ENDOSCOPIC RESECTION FOR RECTAL NEUROENDOCRINE TUMOR}

Ningning Dong*, Peng Li, Ming Ji, Yongjun Wang, Shutian Zhang. Beijing Friendship Hospital, Capital Medical University, China

\subsection{6/gutjnl-2019-IDDFabstracts.206}

Background Endoscopic resection is recommended for rectal neuroendocrine tumors $<1 \mathrm{~cm}$ in diameter; the three techniques (endoscopic submucosal dissection [ESD], endoscopic mucosal resection [EMR], and cap-assisted EMR [EMR-C]) have been previously reported; however, the optimal endoscopic technique remains unknown. In this study, we compared the efficacy and safety of three endoscopic resection methods for rectal neuroendocrine tumor.

Methods We prospectively enrolled 130 patients with rectal neuroendocrine tumors treated by endoscopy at Beijing Friendship Hospital between July 2012 and July 2018. We compared clinical outcomes, including en bloc resection rate, R0 resection rate, procedure time, postoperative complications, local recurrence and distant metastasis, in three groups based on the endoscopic treatment method. 